\title{
Unexpected Hypertensive Pneumothorax after Digestive Upper Endoscopy: A Case Report
}

\author{
Giovanni Zagli $^{1 *}$, Rosario Spina ${ }^{1}$, Stefano Batacchi ${ }^{1}$, Giancarlo Freschi ${ }^{2}$, Manlio Acquafresca ${ }^{3}$, \\ Antonio Taddei ${ }^{2}$, Adriano Peris ${ }^{1}$
}

\begin{abstract}
${ }^{1}$ Anesthesia and Intensive Care Unit of Emergency Department, Careggi Teaching Hospital, Florence, Italy; ${ }^{2}$ Department of Surgical Pathology, University of Florence and Careggi Teaching Hospital, Florence, Italy; ${ }^{3}$ Diagnostic Imaging Department, Careggi Teaching Hospital, Florence, Italy.

Email: "giovanni.zagli@unifi.it
\end{abstract}

Received June 27 ${ }^{\text {th }}$, 2012; revised July 30 ${ }^{\text {th }}, 2012$; accepted August $21^{\text {st }}, 2012$

\begin{abstract}
We report an unexpected massive left pneumothorax at the end of a digestive upper endoscopy without evidences of perforation or airway over-pressure. The possible air passage through a diaphragmatic failing is discussed.
\end{abstract}

Keywords: Upper Endoscopic Procedure; Pneumothorax; Diaphragmatic Failing

\section{Introduction}

Upper endoscopic procedures can cause respiratory failure by several mechanisms, such as pneumomediastinum and pneumothorax by airway over-pressure developing during procedure or, in case of perforation of the proximal digestive tract, through air-passage into the extraluminal surrounding duodenal structures.

\section{Case Description}

A 50 years old man (weight $60 \mathrm{~kg}$, height $175 \mathrm{~cm}$ ) with an history of gastric lymphoma treated 20 years before with total gastrectomy (Billroth 2 gastrectomy), splenectomy and pancreatic tail resection, underwent to a new surgical intervention due to intestinal occlusion was caused by cancer metastasis (T4 stadiation). Patient was surgically treated with total colectomy, ileal and diaphragmatic resection. After one week from surgery, patient underwent to a gastric transit X-ray for the suspicion of perforation in the esophageal-jejunal tract. The examination revealed contrast media leakage in left subdiaphragmatic region (Figure 1, arrow). In consideration of the complex and multiple surgical manipulations and of patient's conditions, surgeons decided to execute an upper endoscopic examination.

The endoscopic procedure was conducted in the operating room, with oropharyngeal mucosal topic anesthesia and with low-dose propofol sedation in spontaneous breathing (patient in left lateral position, oxygen supple-

${ }^{*}$ Corresponding author. mentation at $50 \%$, heart rate $80 \mathrm{bpm}$, mean arterial pressure $85 \mathrm{mmHg}$, peripheral oxygen saturation 100\%, respiratory rate 18 acts per min). No complications occurred during the endoscopy execution, and procedure resulted negative for perforation.

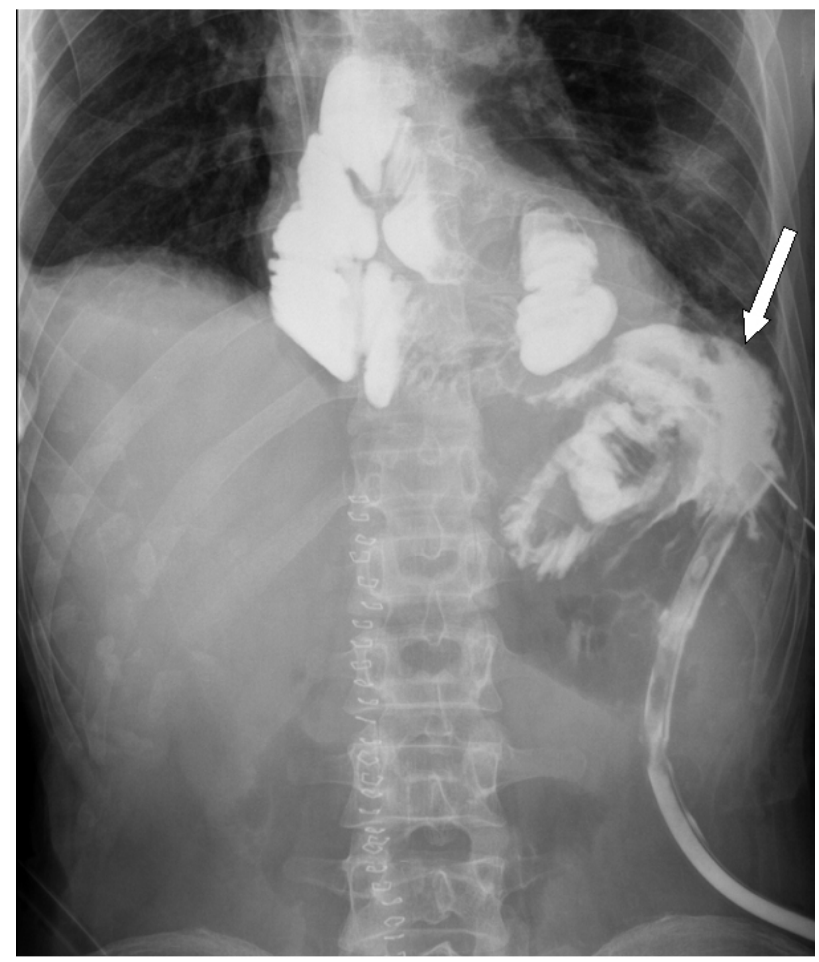

Figure 1. Contrast media leakage in left subdiaphragmatic region (arrow). 


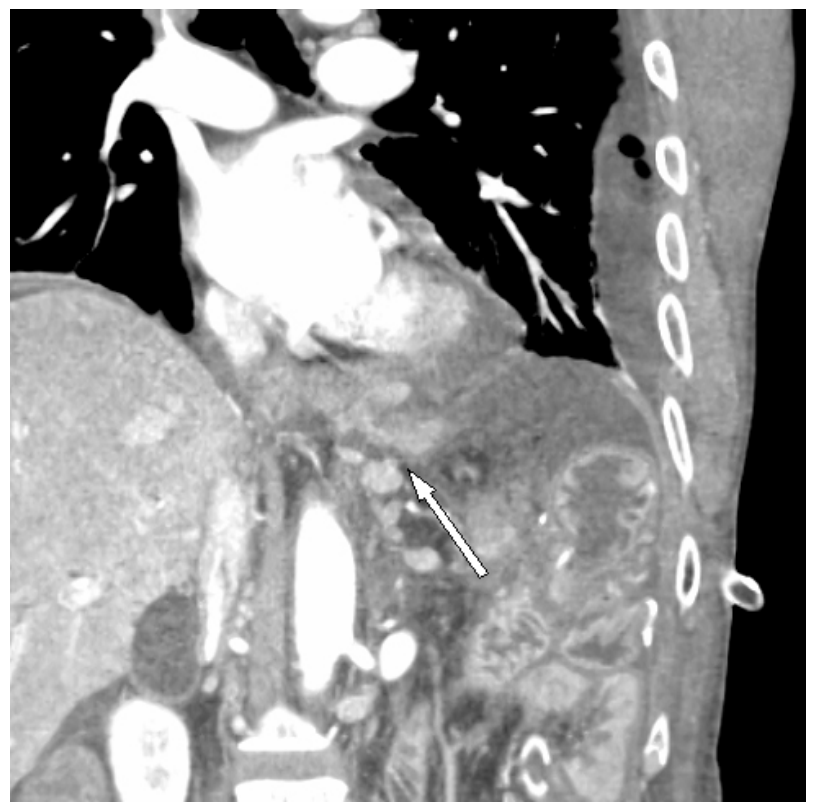

Figure 2. CT scan were the arrow indicates the probable diaphragmatic failing.

At the end of procedure, patient had a sudden respiretory failure with tachycardia (heart rate $>120$, dyspnoea, peripheral oxygen saturation passing from $100 \%$ to $90 \%$ ). The critical episode started immediately after the passage from left lateral position to supine position. Chest auscultation revealed the lack of air flow during ventilatory act on left hemithorax, and bedside chest ultrasound examination confirmed the suspect of left pneumothorax showing absence of pleural gliding and lung movements [1]. A chest tube was rapidly positioned by the anesthesists with air and pleural fluid emission followed by resolution of respiratory distress and disappearance of radiologic signs of pneumothorax. Patient was discharged from the operating room in stable condition. Patient also gave the informed consent for case report publication.

\section{Discussion}

Usually, surgical treatment is required in the management of gastrointestinal leaks. However, patients with a contained leak and complex situations can be feasible managed by endoscopic methods [2]. Upper endoscopy can cause subcutaneous emphysema, pneumomediasti- num and pneumothorax by airway over-pressure developing during procedure [3] or, in case of perforation of the proximal digestive tract, through air-passage into the extra-luminal surrounding duodenal structures and subcutaneous tissue until pleural cavity $[4,5]$. In the present case, no jejunal endoscopic evidences of perforation were detected. As possible mechanism, intraluminal air accumulated during endoscopic phase could have been passed through a diaphragmatic failing (Figure 2, arrow) after the patient position change (from left lateral position to supine position).

\section{Conclusion}

This experience suggests using prudence in treating patients with upper endoscopy in case of recent diaphragmmatic surgical manipulation. Moreover, the possibility to perform bedside chest ultrasound examination might permit a faster diagnosis of pneumothorax in operating room in case of emergency.

\section{REFERENCES}

[1] B. Bouhemad, M. Zhang, Q. Lu and J. J. Rouby, “Clinical Review: Bedside Lung Ultrasound in Critical Care Practice," Critical Care, Vol. 11, 2007, p. 205. doi:10.1186/cc5668

[2] G. S. Raju, "Endoscopic Closure of Gastrointestinal Leaks," American Journal of Gastroenterology, Vol. 104, No. 5, 2009, pp. 1315-1320.

[3] A. Rai and S. Iftikhar, "Tension Pneumothorax Complicating Diagnostic Upper Endoscopy: A Case Report,” American Journal of Gastroenterology, Vol. 94, 1999, pp. 845-847. doi:10.1111/j.1572-0241.1999.00958.x

[4] T. Savides, S. Sherman, B. Kadell, H. Cryer and M. Derezin, "Bilateral Pneumothoraces and Subcutaneous Emphysema after Endoscopic Sphincterotomy,” Gastrointestinal Endoscopy, Vol. 39, No. 6, 1993, pp. 814-817. doi:10.1016/S0016-5107(93)70273-4

[5] A. P. Morley, J. Y. Lau and R. J. Young, “Tension Pneumothorax Complicating a Perforation of a Duodenal Ulcer during ERCP with Endoscopic Sphincterotomy,” Endoscopy, Vol. 29, No. 4, 1997, p. 332. doi:10.1055/s-2007-1004205 